\title{
Romanzi di deformazione. Comunicazioni d'autore e scrittori di massa
}

Ronald De Rooy, Beniamino Mirisola e Viva Paci

\section{OpenEdition}

\section{Journals}

Edizione digitale

URL: http://journals.openedition.org/cei/187

DOI: $10.4000 /$ cei.187

ISSN: 2260-779X

\section{Editore}

UGA Éditions/Université Grenoble Alpes

\section{Edizione cartacea}

Data di pubblicazione: 15 juin 2010

Paginazione: 225-243

ISBN: 978-2-84310-168-7

ISSN: 1770-9571

Notizia bibliografica digitale

Ronald De Rooy, Beniamino Mirisola e Viva Paci, «Romanzi di deformazione. Comunicazioni d'autore e scrittori di massa», Cahiers d'études italiennes [Online], 11 | 2010, online dal 15 décembre 2011, consultato il 26 mars 2021. URL: http://journals.openedition.org/cei/187 ; DOl: https://doi.org/ $10.4000 /$ cei. 187 


\title{
ROMANZI DI DEFORMAZIONE. COMUNICAZIONI D'AUTORE E SCRITTORI DI MASSA
}

\author{
Ronald De Rooy \\ Université d'Amsterdam \\ Beniamino Mirisola \\ Université Ca’ Foscari - Venise \\ Viva Paci \\ Université de Montréal
}

Questa comunicazione rispecchia alcune linee del progetto di ricerca a cui stiamo attualmente lavorando: Romanzi di deformazione (e altre piste di una generazione di narratori italiani-1998-2008).

Seguendo le tracce di quegli scrittori che sembrano aver meglio disegnato i contorni della nostra epoca e del nostro Paese, ci avventureremo nello scenario magmatico e ipertecnologico che fa da sfondo privilegiato alle loro narrazioni. Un territorio, questo, dove alla mitologia tradizionale ne è subentrata una che attinge all'immaginario pubblicitario e a quello dei videogames. Un territorio dove risuona uno slang televisivo che, giorno dopo giorno, perde sempre più terreno, a favore degli idioletti dei nuovi e più agguerriti mass media. Un territorio, infine, dove i personaggi si muovono confusi, spaesati, privi ormai di quelle coordinate che possano consentire loro una, seppur approssimativa, lettura del mondo. 


\title{
DEFORMAZIONI FAMILIARI E MEDIATICHE NELLA NARRATIVA ITALIANA DI OGGI
}

\author{
Ronald De Rooy
}

A partire dalle dediche e le epigrafi, i romanzi esaminati in questo contributo si iscrivono subito in un discorso familiare. Sandro Veronesi dedica Caos calmo (CC) "Ai miei figli» e Melania Mazzucco per Un giorno perfetto cita parole significative dal Discorso sullo stato dell'Unione, nel 2004, di George W. Bush: «La famiglia è il luogo in cui dimorano le speranze del nostro paese, il luogo che fa spuntare le ali ai sogni» (GP, p. 7). Si tratta di elementi paratestuali che vanno letti antifrasticamente, perché gli intrecci di questi romanzi propongono della famiglia un'immagine problematica, disperata, deformata.

Nel breve tempo a mia disposizione vorrei cercare di gettare un po' di luce su due temi fondamentali che ricorrono in questi romanzi. Primo: la deformazione della famiglia e in particolare quella della figura del padre e di quella dei figli. Secondo: l'effetto dei media e della rivoluzione digitale su questa deformazione familiare.

\section{La famiglia deformata e il ruolo negativo del padre}

È chiaro ormai che la tensione tra famiglia e individuo è presente in quasi ogni romanzo di Veronesi. Dopo La forza del passato (FP), tutto concentrato sull'enigmatico fantasma del padre morto, Caos calmo si focalizza su una famiglia in cui la madre è venuta a mancare improvvisamente. Una famiglia decapitata, composta da un padre di quarantatré anni e da una figlia di dieci. Come La forza del passato, anche Caos calmo analizza prevalentemente la psicologia del padre. Pietro Paladini costituisce il centro d'orientamento dell'intero romanzo, in quanto vedovo ma soprattutto in quanto padre.

A tanti osservatori esterni, infatti, Pietro sembra un padre straordinario che si sacrifica completamente per alleviare il dolore di sua figlia 
- «Wow. Che padre» è la reazione emblematica della bellissima Jolanda (CC, p. 400). Può sembrare, infatti, che Pietro rimuova nobilmente la propria sofferenza dedicandosi interamente alla figlia, dal momento che decide di non tornare più in ufficio ma di sorvegliare fedelmente davanti alla sua scuola.

D'altra parte, non possiamo nemmeno escludere che la perdita di sua moglie non lo faccia soffrire più di tanto. E anche nel ruolo di padre Pietro si dimostra sempre più inadeguato e insensibile. Anzi, dietro l'apparenza paterna e premurosa si cela un uomo che pensa soprattutto a se stesso. Molti lo spingono nel ruolo di padre, ma lui quasi non se ne accorge. Infatti, la figura del padre serpeggia ossessivamente nelle conversazioni con i suoi parenti e colleghi.

La megafusione che è in corso nella azienda di Pietro spinge tanti colleghi e padroni a venire da lui per scaricargli addosso il loro dolore (CC, p. I07), per «vomitare [...] le proprie preoccupazioni» (CC, p. I46). E tutti tendono a vedere in Pietro il padre modello, il padre per eccellenza, quel padre che loro non hanno mai avuto.

Il super-manager Jean-Claude lo paragona implicitamente al proprio padre che non gli avrebbe mai dedicato tanto tempo: «era pilota di caccia militari $[. .$.$] e, forse, un agente segreto. Era sempre via. Non è mai venuto$ a prendermi a scuola, nemmeno una volta» (CC, p. 65). A parte l'interessante allusione al padre misterioso di La forza del passato, si coglie il dolore dell'uomo adulto che durante una crisi professionale significativamente rivive il trauma infantile del padre assente. Nella fase conclusiva del romanzo lo stesso Jean-Claude, ubriaco fradicio, telefona a Pietro nel mezzo della notte per dirgli che il comportamento indifferente di suo padre era perfettamente comprensibile: faceva il pilota, era in realtà un agente segreto e per questo non veniva mai a prenderlo a scuola. «Non poteva, capisci?», «Però mi voleva bene» (CC, p. 329).

Paolo Enoch, il capo dell'Ufficio Personale, confida a Pietro che voleva diventare sindacalista, come suo padre. Ora che la fusione terrorizza tutti non gli piace più il suo ruolo nell'azienda e pensa spesso a suo padre, a come lui si sarebbe comportato al suo posto. La scelta di dimettersi e di raggiungere suo fratello missionario nello Zimbabwe segue direttamente da questo confronto.

Last, but certainly not least, c'è l'apparizione del Padre trinitario nell'allegoria che Paolo Enoch applica alla megafusione. Enoch spiega che nella fusione dei due grandi gruppi industriali si adotta un modello sbagliato. L'ebreo Steiner è il capo del gruppo americano, il cattolico Boesson guida il gruppo europeo. Dopo la fusione Boesson diventerà «Dio in terra, pédégé 
del più grande gruppo di telecomunicazioni nel mondo, sarà il Dio del suo nemico» (CC, p. 269). Una posizione insopportabile, come Boesson confermerà più tardi. Tutto a causa del fatto che non si è scelto «il canone cattolico», «elastico e complesso» (CC, p. 268), cioè la Trinità, «Padre, Figlio e Spirito Santo» (CC, p. 269). Si è preferito invece il canone ebraico "gerarchico e immutabile» (CC, p. 268) che prevede «il Dio vecchio e solitario» sullo «scranno più alto di tutti». Ci volevano invece «tre scranni alla stessa altezza: uno per lo Spirito Santo [...] uno per il Padre e uno per il Figlio» (CC, p. 269). Nel contesto aziendale Pietro intende perfettamente questo ragionamento ed avrà modo di esporlo con successo a Boesson, colui che sta per diventare il Padreterno onnipotente della nuova mega-azienda.

Quello che Pietro non capisce, però, è che questa filosofia aziendale potrebbe essere anche una chiave per la situazione personale e familiare di Pietro stesso. La fusione di aziende è paragonata implicitamente alla creazione di una famiglia e la creazione di una famiglia ad una fusione di aziende. Pietro e Lara avevano creato una famiglia, ma con la morte di Lara la loro famiglia si è deformata, si è rotto l'equilibrio trinitario, e Pietro si è visto improvvisamente nel ruolo del Dio solitario, un ruolo che non riesce a sostenere. $\mathrm{Ci}$ vorrà l'iniziativa della Figlia minorenne per indicare la strada al Padre smarrito. Appena decenne, Claudia si dimostra stranamente adulta quando deblocca la paralisi del padre indicandogli di riprendere la sua vita: «Devi mettere ordine nella tua vita, darle un verso, un senso, perché il caos che governa quella dei bambini è bello, certo, ma tu sei un uomo.» (CC, p. 45I.)

Non raramente questa nuova generazione occupa un posto interessante nella recente narrativa italiana. Da una parte i giovani sono "per definizione innocenti, in un mondo per definizione corrotto». Assumono il ruolo di vittime, "vittime degli adulti [...], della società, [...] della pedagogia» (Sim., p. 75). Dall'altra parte, però, colpisce sempre più spesso la maturità di questi ragazzi rispetto agli adulti che li circondano e che dovrebbero guidarli, educarli, e confortarli. In Caos calmo il padre emerge come una figura oppressiva o come una figura disorientata che non sa più cosa fare del suo potere. La Figlia, invece, rappresenta nel migliore dei casi una possibile liberazione, una creatività leggera di "caos calmo» che infrange la legge del padre.

Con Un giorno perfetto Melania Mazzucco offre una visione pluriprospettica della vita familiare e sociale nell'Italia di oggi. Solo qualche rapida osservazione. La narrazione molteplice è tessuta intorno a tre famiglie. C’è la ricca famiglia altoborghese Fioravanti, apparentemente unitaria, ma in realtà squilibrata e fredda. C'è la famiglia borghese Buonocuore, dove la 
devastazione è spaventosa e la famiglia è diventata un luogo di violenza, psicologica e fisica, una famiglia deformata che sta per soccombere definitivamente. E finalmente, c'è la famiglia alternativa irraggiungibile, ma sognata ardentemente dall'insegnante omosessuale Sasha.

Anche in questo romanzo la figura problematica su cui si concentra il male è il padre. Riconosciamo un Pietro Paladini nel ricco ed abile politico Elio Fioravanti, un uomo nel pieno di una crisi professionale, ma anche un padre che scopre di non saper gestire la famiglia, l'educazione dei suoi figli. Come Pietro Paladini anche lui avrà paradossalmente bisogno di essere guidato dalla sua bambina. Diversamente da Paladini, però, Fioravanti non è in grado di cogliere i segnali della bambina.

Il focus narrativo principale è, invece, su Antonio Buonocuore, un padre totalmente sbandato. Dopo la separazione dalla sua famiglia Antonio oscilla tra dolore e rabbia impotente. In preda a pensieri di vendetta contro la moglie egli giustifica le terribili azioni che sta per compiere con disperati riferimenti alla Scrittura. La sua solitudine non gli permette di uscire dal circolo vizioso della rabbia e anche lui non è capace di sintonizzarsi con i messaggi vitali dei suoi figli. Antonio continua a credere a modo suo nella famiglia, scrivendo nella sua lettera di congedo: «la famiglia è lo scopo più nobile della vita di un uomo che altrimenti pesa sulla terra come un sasso senza lasciare frutto e discendenza» (GP, p. I78); l'unica famiglia che conosce, però, è quella dominata dal padre.

\section{Effetti deformanti di tecnologia e media di massa}

Com'è stato notato ormai da molti critici (per esempio Sin. e Raj.), i media, quelli relativamente antichi come cinema, televisione e pubblicità e quelli più recenti come internet, e-mail, videogiochi eccetera, si sono inseriti ed intimamente fusi nella narrativa postmoderna. I modi narrativi sono diventati intermediali, i mondi narrativi hanno acquistato un carattere intensamente mediatico. Negli anni Novanta, questa intermedialità aveva spesso un carattere forte ed esibito, complementato da un appiattimento fumettistico dei personaggi, dall'universalizzazione dello spazio, e da trame grottesche e spesso molto violente. Nell'ultimo decennio, invece, da varie parti si è parlato di fine del postmodernismo (Lup.), di neomodernismo, di realismo critico (Sim. e Cas.), un ritorno, insomma, a forme di realismo moderato. In questo ritorno a modi narrativi più realistici la intermedialità non è scomparsa, ma essa risulta più "spontanea", più naturale, i personaggi sono in genere meno piatti, più realistici, e la violenza più 
funzionale al racconto. Raffaele Donnarumma afferma a questo proposito: «messa da parte l'ubriacatura testualizzante, sembra infatti che si possa registrare un ritorno critico al realismo come indagine sui conflitti interni alla società occidentale» (Sim., p. 79). Questa affermazione può applicarsi anche alle narrazioni su famiglie deformate qui esaminate, e in particolare alla loro rappresentazione della rivoluzione digitale e massmediatica. Qual è precisamente il ruolo dei media di massa in questi romanzi? La rivoluzione digitale ha forse acuito anche in questi contesti letterari il gap generazionale tra padri e figli?

Cominciamo da Caos calmo. Pietro Paladini possiede tutto quello che c'è da desiderare nella vita moderna. Infatti, all'inizio della sua narrazione egli riassume il successo della sua vita con un elenco di oggetti tecnologici e di lusso: macchina, case, telefonino eccetera: «Possiedo una bella casa nel centro di Milano, un bastardo di fox-terrier di nome Dylan, un'altra bella casa al mare, in Maremma, a metà con mio fratello Carlo, e un'Audi A6 3000 Avant nera piena di optional costosissimi» (tra cui il giocattolo del gps) (CC, p. 27). Anche nel seguito Pietro si dimostra molto attaccato alle cose. Quando il suo simpatico collega Enoch lascia la ditta e il paese per lo Zimbabwe, Pietro assiste a bocca aperta a quella che chiama significativamente la «spoliazione dell'uomo occidentale»: «Casa, azioni, macchina, telefonino» (CC, p. 273). Per Enoch si tratta di zavorra inutile, ma l'avido Pietro non resiste al fascino della ricchezza illimitata che vede ad esempio nei suoi super-padroni. Di volta in volta egli cede facilmente alle tentazioni di questo mondo leggero e apparentemente perfetto. Quando Thierry gli propone di prendere il posto di Jean-Claude, Pietro vede spiattellati davanti a sé tutti $\mathrm{i}$ "frutti più succulenti che la civiltà alla quale appartiene sia in grado di offrire» (CC, p. I65). Anche nell'importante colloquio con Boesson Pietro si vede ben volentieri nel ruolo lucroso dello Spirito Santo: anche lui «un titano, un dio» (CC, p. 432). E per un attimo s'immagina nuovamente l'incredibile ricchezza e la conseguente felicità che questa nuova posizione comporterebbe.

Nell'universo manageriale e materiale di Pietro gli oggetti di lusso e i gadget elettronici servono a definire l'identità dei loro proprietari. Mi limito ad una brevissima rassegna. Al super-manager Jean-Claude hanno tolto l'aereo, il suo "super-giocattolo", e questo evento produce in lui «lo stesso dolore pazzo e insopportabile» che prova un bambino quando gli si strappa di mano il Game-boy (CC, p. 67). Non essere al passo con i tempi digitali equivale ad esporsi ad imbarazzo e derisione. Il topmanager Piquet si compra un notebook iperavanzato ma disgraziatamente scambia il vassoio $\mathrm{cd} / \mathrm{dvd}$ per un porta-lattine estraibile. Dopo questa gaffe al pove- 
raccio non resta che scappare via e scomparire: «cellulare spento, segreteria di casa staccata, niente» (CC, p. 374). La decenne Claudia riceve dallo zio Carlo un videofonino, un oggetto che le dà tanta e tale gioia da provocare un'atroce gelosia nel padre: toccava a lui darle un simile regalo. E un telefonino uguale a quello di Carlo stesso e, guarda caso, anche a quello che Enoch aveva lasciato su un cassonetto: un oggetto, che in quell'occasione era «lasciato lì a rappresentare tutto il male del mondo» (CC, p. 278), ma Pietro continua a rodersi per l'incapacità di controllare e di esaudire i desideri high tech di sua figlia.

In Un giorno perfetto, vero e proprio romanzo di vite vissute in real time, i figli sono decisamente le vittime degli adulti, ma in qualche modo anche dei media di massa e della rivoluzione digitale. In particolare gli adolescenti si chiudono nei loro microuniversi digitalizzati e multimediali. Con il suo walkman Valentina vive solipsisticamente in un mondo dove regna lo shockrock di Marilyn Manson, le cui parole provocatorie, e sinistramente profetiche, lei canta in un autobus affollato: "Flies are waiting / in the shadow / of the Valley of Death [...] Some of us, are really born to die», sognando nel frattempo "Un mondo senza autobus, senza Roma, senza mamma - un mondo di musica. SWEET DREAMS ARE $M A D E$ OF THIS» (GP, p. 72). L'assenza di comunicazione tra madre e figlia in questi momenti è totale: «La musica fracassona che sgorgava dagli auricolari del suo walkman la teneva a distanza» (GP, p. 73). Per liberarsi dalle domande della madre Valentina prende il suo cellulare e comincia a controllare messaggi e mandare sms. Anche il cellulare diventa così uno strumento di alienazione, che le permette di fuggire in un mondo che $\mathrm{i}$ genitori non sanno capire. La madre Emma sente tutto il peso dell'insormontabile abisso tra lei e i suoi figli:

Gli unici momenti in cui li aveva tutti e due accanto - momenti preziosi, eppure inutilizzabili - resi muti dal fragore del treno, storditi dal calore malsano dei vagoni, pigiati fra estranei, senza potersi parlare, senza ascoltarsi, vicini ma in realtà lontani, assenti. Altrove. Sto perdendo i loro momenti più belli. E non torneranno. (GP, p. 79.)

Da parte sua, anche Valentina è lontanissima dal capire i suoi genitori. Paradossalmente le sembrano più vicini e più accessibili gli universi e i personaggi del cinema:

Capisco di più ET o l'androide Roy di Blade Runner che le persone che mi stanno vicino. Mi sento più simile a loro che a mia madre o a mio padre. Non capisco i sentimenti connessi alle relazioni personali tra gli esseri umani. Non capisco perché si amano, cosa li spinge a farsi male gli uni con gli altri, come possano odiarsi tanto. Forse non provo le emozioni. O forse non so cosa è essere umano. (GP, p. 325.) 
Anche ai bambini più piccoli il mondo virtuale offre qualche consolazione superficiale e qualche ingannevole possibilità di fuga da una realtà crudele. Così, il piccolo Kevin usa ad esempio il ritornello dei Digimon ("Digimon, Digital Monsters / Digimon are the champions», GP, p. 87) come un mantra taumaturgico per sopravvivere momenti di imbarazzo ed umiliazione. Durante le ultime ore della sua vita, Kevin s'immerge nel Re Leone di Walt Disney, commuovendosi e piangendo per l'ennesima volta per la scena in cui il padre Mufasa muore sotto gli occhi del cucciolo Simba. Nel frattempo sua sorella riesce a mandare un ultimo sms per rassicurare la madre: «CI DIVERTIAMO CON PAPÀ TUTTO OK MANKI SOLO TU BUONANOTTE MAMMA TVTB!!!» (GP, p. 359.) Così, i media di massa e le tecnologie aumentano la distanza tra i familiari e aggravano il loro senso di alienazione e solitudine. La scena strappalacrime del Re Leone si riflette nella morte vera del piccolo Kevin per mano del proprio padre. E l'ultimo sms di Valentina offre a sua madre una rassicurazione totalmente inaffidabile. Ma la deformazione mediatica più terribile la troviamo nella frenesia di Antonio, che prima di uccidere sogna di poter filmare gli omicidi e così di moltiplicare infinitamente la sofferenza della moglie:

Agiva come se Emma fosse la spettatrice del film che andava girando. Il film della loro vita, nel quale lei aveva cercato di ridurlo a una comparsa insignificante - mentre era, e sarebbe rimasto per sempre, il protagonista. Gli verrebbe in mente che avrebbe potuto davvero filmare la scena, e allora lui e i bambini sarebbero morti milioni di volte. (GP, pp. 365-366.) 


\title{
«SENTI CHE BEL RUMORE...»
}

\author{
Viva Paci
}

Non è domanda inedita, ma è pertinente nel cuore della letteratura dei nostri giorni: cosa sa e cosa può farsene un testo letterario di altri linguaggi mediatici? Alcuni romanzi recenti, Come Dio comanda (CDC), Amore mio infinito (AMI) e Mi chiamo Roberta, ho 40 anni, guadagno 250 euro al mese (MCR), appaiono degli esempi egregi di linguaggi mediatici riassorbiti nel testo letterario: ci aiuteranno allora a proporre qualche riflessione sulla questione della ri-mediatizzazione (remédiation) nella produzione narrativa italiana contemporanea.

Riprendo i sentieri aperti da Ronald de Rooy, nella sezione precedente, attenta soprattutto a questioni di contenuto narrativo, mi concentro su altri due scrittori che disegnano i contorni della nostra epoca, e del nostro Paese, con invidiabile intelligenza e ironia: Niccolò Ammaniti (classe '66) e Aldo Nove (classe '67).

L'idea è semplice: non si tratta più, con i nostri autori, di collage postmoderni - in cui al lettore è dato di identificare le parti eterogenee, le soluzioni di continuità, come era il caso, per esempio, di Blackout di Nanni Balestrini (Bal., 1980) - o di uno schieramento dei giorni nostri tra gli echi degli apocalittici o degli integrati (Eco). Bensì, nelle pagine scorrevoli e lineari dei loro romanzi, si presenta un'interessante remédiation di alcune forme proprie ad altri media - di massa - e si gioca, laddove non si crederebbe a priori, la resistenza dell'Autore. Pagine che ho definito scorrevoli, come una cantilena, un monologo interiore - nel caso di Nove - e, comunque sia, un vero e proprio assedio dell'oralità nel testo scritto - nel caso di Ammaniti; e lineari, nel senso che presentano, tutto sommato, scorie extra-letterarie meno imponenti, rispetto a quelle analizzate a proposito di Caos Calmo nelle pagine che precedono e di King of Kings in quelle che seguiranno.

Due le questioni evocate da trattare: il ruolo paradossale dell'autore, e il lavoro di linguaggi non letterari nel cuore della letteratura. 


\section{«Lo chieda al mio manager letterario»}

Senza entrare nel vivo della questione della "morte dell'autore" - questione teorica troppo vasta, da Foucault a Eco... -, due tipi di apparenti sparizioni dell'autore si mettono in scena nei due romanzi qui presi in esame.

Eletti a oggetto di culto, vere rockstar della letteratura, nel bene e nel male, Ammaniti e Nove (il primo più del secondo), mettono - forse proprio per questo - alla prova la posizione dell'autore. Esempi paratestuali, che si possono rintracciare nelle strategie editoriali, ce lo provano: le scuderie Mondadori possono permettersi di puntare talmente su Ammaniti che lo fanno sparire dalla copertina. L'esempio di Come Dio comanda sembra dimostrare, già dall'illustrazione di copertina, quanto l'autore sia considerato dal suo editore un "cavallo da corsa" su cui puntare. Infatti, il progetto grafico della copertina rigida non mostra alcun carattere scritto sulla facciata (Art Director: Giacomo Gallo, progetto grafico: Cristiano Guerri). Un lampo squarcia il nero-blu di una notte di tempesta. È tutto. Niente titolo, niente autore. Una sola sovracopertina trasparente su cui i caratteri stampati in bianco appaiono appena visibili, con il nome dell'autore e il titolo. Si sottintende, dunque, che se sparisse questo foglio, questo peritesto effimero, l'autorialità del romanzo si manifesterebbe altrimenti, sopravvivendo anche senza il nome dell'autore in copertina.

Einaudi, dal canto suo, per Nove si lancia tranquillamente nella pubblicazione della sua opera recente, Mi chiamo Roberta, ho 40 anni, guadagno 250 euro al mese, in una collana di scienze politiche. Ciò che Einaudi pubblica è, in fondo, una raccolta di interviste a persone vere, normali - ma normali davvero, non come i "normali" di Superwoobinda (SW), che poi si trasformano in zombie spettacolari -, individui privati e non celebrità, e per di più interviste già apparse nella loro veste mediatica più consona, pubblicate cioè una per una, sui quotidiani.

Dal fenomeno mediatico Ammaniti, poi, prende avvio una strategia nascente nell'editoria italiana, in sintonia con le palazzine molto cool e branchées della Mondadori Media Store: quella del Book Trailer. Veri e propri video-clip che presentano un romanzo, azzardando finanche letture e interpretazioni sul senso dell'opera. Dallo stile raffinato, in relazione continua con un immaginario anche cinematografico, nel Book Trailer di Come Dio comanda si riconosce una chiara ripresa stilistica del David Lynch di Twin Peaks - autore familiare all'universo visivo di Ammaniti, peraltro: si pensi ad Io non ho paura (IN), romanzo del 200I, ma anche al film omonimo di Salvatores, del 2003. 
Siamo, insomma, nella sfera di una vera e propria creazione di mythologies contemporanee: e la politique des auteurs, per quanto riguarda $\mathrm{i}$ Nostri, oltrepassa le divisioni tra le discipline, tra i linguaggi e tra i media, in una irradiazione in altri media e di altri media, che provoca una sorta di proliferazione di prodotti derivati.

Irradiazione in vari sensi, in varie direzioni: tanto il reportage entra nel libro dell'Autore, quanto l'Autore porta la sua creazione nelle pagine del quotidiano; tanto l'Autore porta l'immaginario del cinema e il linguaggio del montaggio audiovisivo nel suo romanzo, quanto il romanzo stesso infiltra la rete.

Conseguenza ne è questa idea di prodotto derivato, in cui poi non si è più sicuri di cosa sia derivato da cosa: un fenomeno di mercato che mi pare, in fondo, ispirato alle nuove strategie di marketing delle grandi campagne pubblicitarie.

\section{«Come l'autore nell'Atelier du peintre»}

"The Waste Land de T. S. Eliot est une fresque à cinq dimensions parsemée de papiers collés qui éprouvent la solidité de l'édifice [où] l'auteur est aussi présent que Courbet dans L'atelier du peintre."

Pierre Leyris, traducteur d'Eliot.

Vediamo ora più da vicino, in un abbozzo di analisi intermediale, come in due esempi diversi ma sintomatici (Amore mio infinito e Come Dio comanda) si metta in scena, meglio in pagina, una relazione tra alcuni frammenti dei linguaggi di altri media e la struttura narrativa letteraria.

Alla lettura, in Amore mio infinito, del "Prologo. Ottobre 2000» un dato è chiaro: stiamo entrando in una brochure che canta le lodi di un prodotto da vendere. Il linguaggio utilizzato è dichiaratamente promozionale, pura retorica industriale e pubblicitaria: Matteo parla, infatti, come il catalogo della ditta di banconi commerciali per la quale lavora. Ci sono anche delle ragioni che possiamo definire "narrative" per questo esercizio di stile: il protagonista, appena trentenne, è ormai stato fagocitato dal sistema; prima aveva una voce e una vita [qui è caduta una parola: vita?] proprie, ora non più, e noi impareremo a conoscerlo, dal primo al 
quarto capitolo (che poi, per seguire il flusso della coscienza del personagionarratore, si chiamano: prima, seconda, terza e quarta cosa), sempre indaffarato con l'amore.

Un altro esempio di ri-mediazione è quella giornalistica/radiofonica/ da reportage televisivo, che Nove compone nella sezione La violenza illustrata 1976: un vero e proprio remix di La violenza illustrata di Balestrini (Bal., 200I).

Al di fuori di questi due diversi inserti di altri linguaggi, la questione del frammento ha a che fare con tutta l'opera di Nove, ed è lì che si trova una relazione importante, a mio avviso, con i media di massa - e non tanto perché Nove parla compulsivamente dei media di massa. La lingua che usa evoca il balbettio, il singhiozzo e la sgrammaticatura - è ovvio - di un racconto in prima persona; e qui l'altro filo teso tra Nove e Balestrini: si pensi alle età di Matteo in Amore mio infinito e alla sua lingua che cambia e, ovviamente, a Superwoobinda e li si confronti con il Balestrini di Vogliamo tutto (Bal., 197I) e di I Furiosi (Bal., I994).

In Nove, però, più che di quel discorso libero indiretto che è stato riconosciuto come la cifra stilistica dei citati esempi di Balestrini, siamo in presenza di un'organizzazione formale che attinge ad una rappresentazione mediata da una telecamera che filma e registra l'audio del personaggio, introducendo un chiaro zapping letterario: un'organizzazione, anche visiva, della pagina che rinvia ad un'operazione di vero e proprio taglia e incolla dal giornale. Prima di Word, ci ricorda Gian Paolo Renello (Ren.), era stato Burroughs ad aver fatto ricorso alle tecniche del cut up e del fold in, marcando, anche per questo, la letteratura beat americana.

L'impressione della composizione per frammenti è forte - da qui, la tentazione legittima di parlare di tecnica del montaggio. Il che non è, certo, una novità: elemento già forte nelle avanguardie storiche, in Balestrini rappresentò una vera e proprio pratica (giornali, conversazioni, altre opere letterarie). Nove la mima e la ricostruisce (si pensi al caso del remix accennato), Ammaniti la formalizza.

Il riferimento all'uso della telecamera ha una precisa implicazione per quanto riguarda questa costruzione per frammenti (e non si è usato qui telecamera come si sarebbe potuto utilizzare macchina da presa: la differenza tecnica ne implica, per noi, una semantica). Se pensiamo alla costruzione formale di Come Dio comanda, che procede appunto per frammenti, ognuno dei quali focalizza le azioni individuali dei personaggi della comunità di cui si racconta, siamo portati a parlare di uno sviluppo in montaggio alternato delle varie vicende. Ma di che montaggio alternato si tratterebbe? In realtà, i raccordi tra un'azione di un personaggio e 
un'altra sarebbero tutti falsi. Faux raccords, jump cuts: quando il punto di vista cambia, le azioni non sono consecutive, ma si accavallano. Il che suscita una continua impressione di simultaneità: e la simultaneità non è affare del cinema, anzi è la sua bestia nera.

Negli studi sull'archeologia della televisione, si tende a spiegare la potenza di questo medium e il terreno che ha, così in fretta, guadagnato sul cinema, proprio grazie alla forza con cui è riuscita a soddisfare il desiderio di simultaneità: vedere tutto, vedere tutto insieme e vederlo subito (Ur.). Ciò a cui assiste il lettore-spettatore di Come Dio comanda è una forma di montaggio non lineare, in vivo, dove si ha accesso alla simultaneità: quasi fosse lo sguardo di Dio, oppure un colpo d'occhio su una di quelle centraline di montaggio degli studi televisivi, con quindici monitor, in uscita dalle quindici telecamere sul set.

Non sarà forse che il linguaggio dei media visivi entra davvero nella letteratura italiana proprio adesso, quando ormai non è più quello puro del cinema, ma quello già rimediato da altre forme visive, in particolare dalla televisione? Nella nostra osservazione sulla letteratura contemporanea, attuale, popolare, questa considerazione sembra prendere peso. 


\title{
FORWARD THIS MESSAGE: LA NARRATIVA \\ DI STEFANO MASSARON
}

\author{
Beniamino Mirisola
}

Se è innegabile che, tanto sul piano tematico che su quello linguistico, la televisione costituisca un referente imprescindibile per molti dei narratori italiani che hanno esordito negli anni Novanta - valga per tutti il nome di Aldo Nove - la prospettiva muta alquanto se si prende in esame l'opera di Stefano Massaron. Consulente editoriale, redattore di Rolling Stones, traduttore di autori quali Jonathan Coe, Jeffery Deaver e Joe R. Lansdale, lo scrittore milanese è coetaneo dello stesso Nove, nonché partecipe della medesima temperie culturale. Come lui, ha preso parte, nel '96, alla fortunata esperienza di Gioventù Cannibale, ma lo ha fatto in modo del tutto anomalo, scegliendo l'ottica di un buon padre di famiglia che sta «finendo di pagare il mutuo della casa, un appartamentino di tre locali più servizi in un quartiere relativamente tranquillo di Milano» ( $\mathrm{Rm}, \mathrm{p}$. I25). La storia che questi rievoca, a quasi quarant'anni di distanza, non è priva di violenza e di scene cruente, ma sembrano messi da parte i crismi più riconoscibili dell'antologia, quegli stessi che pure avevano caratterizzato buona parte della sua narrativa precedente, dalla Prole immonda (PI) a Lezioni notturne (LN). Manca qui ogni forma di sarcasmo, di vis iconoclasta o di sfida alla verosimiglianza; viene meno il consueto repertorio d'immagini e di moduli espressivi tratti da quella musica e da quel cinema che una certa consuetudine ha, piuttosto sbrigativamente, eletto a simbolo di un'intera generazione; si registra, infine, qualche significativa assenza: i giovani, i prodotti e gli scenari della civiltà post-industriale, i generi di largo consumo, i marchi registrati e... la televisione.

Essa non manca in altri luoghi della produzione massaroniana, ma il suo ruolo appare piuttosto da comprimario che da protagonista, piegato com'è a quello di semplice schermo, quando non di più o meno prezioso oggetto d'arredo. Verrebbe allora da pensare alle parole di Giorgio Gaber, che invitavano a trattarla «[...] in tutte le famiglie/con lo stesso rispetto che è giusto avere/per una lavastoviglie»(Gab.). Invece l'atmosfera è del 
tutto diversa: Massaron ci trasporta in un territorio da cui ogni forma di buon senso è stata ormai bandita e dove l'elegante ironia di Gaber suonerebbe come un malinconico anacronismo. Il medium televisivo ha fatto sì un passo indietro, ma solo per offrirsi quale supporto tecnico ad altre Nuove Ossessioni, che risultano maggiormente insidiose, perché non se ne sono ancora definite le coordinate né sviluppati degli efficaci anticorpi.

Tra queste, un posto privilegiato è riservato ai videogames, autentico tòpos massaroniano, nonché passione dichiarata dello stesso autore, il quale, avvalendosi dell'ausilio di grafici e di programmatori, realizza nel '98 un romanzo, dal titolo Graffiti, interamente costruito intorno alla Playstation, tanto dal punto di vista tematico che da quello strutturale: a ogni capitolo corrisponde, infatti, un gioco e a ogni paragrafo un livello. Ulteriori suddivisioni interne vengono indicate con il segno "@”, a testimoniare - insieme a ulteriori elementi, quali il ringraziamento alla mailing list - una relativamente precoce attenzione per i vari aspetti del web.

Se questo insolito libro, che si chiude sulle parole «è ora possibile spegnere il computer» (Gr, p. I03), inneggia a Lara Croft - nota icona dei giochi telematici, che ritornerà in Gioia della Playstation (KK, pp. 33-37) come mito collettivo, in altri casi è invece il mito personale a essere celebrato attraverso uno schermo televisivo svuotato di pubblicità e di palinsesti. Esemplare, in tal senso, appare il racconto Zoom, contenuto in Lezioni notturne, dove un individuo del tutto amorale, che ha "trovato nel narcisismo il segreto dell'equilibrio mentale» (LN, p. I26), assiste estasiato allo scorrere della VHS su cui ha immortalato i propri amplessi, i propri atti di sadismo e gli omicidi delle proprie amanti. Sulla stessa linea, King of Kings mette in scena un giovane di nome Stefano Massaron che filma i suoi rituali autolesionistici, a base di lamette e di altri oggetti quotidiani utilizzati come strumenti di tortura, per poi godersi la visione in «qualità digitale, sedici noni in standard» (KK, p. 84) sul suo Philips WideScreen.

L'apparecchio televisivo diventa, dunque, anche qui riflesso privilegiato di una visione deviata e sovente disumana della realtà, ma ha cessato di essere il contenitore delle nevrosi e dei sogni collettivi: ad assolvere a una simile funzione è ora la pagina scritta. Una pagina forzata e deformata fino al parossismo, attraverso l'inclusione di e-mail, sms, emoticons, schermate di computer, display di videoregistratori e di bancomat, formule chimiche e trascrizioni di sedute d'analisi, solo per fare qualche esempio. Questa messe di materiale extraletterario - particolarmente copiosa nel romanzo per ragazzi Doppioclic (Dc), pubblicato dalla Disney nel 200I - viene sempre riportata nella sua forma originaria, o in una che le si avvicini il più possibile, in modo che risulti minimo lo sforzo per adeguarsi al testo 
tradizionale e che sia quest'ultimo a doversi espandere o comprimere, o comunque dislocare, fino a perdere quasi del tutto i propri connotati.

Pur nella consapevolezza che un esperimento del genere si rifa a ben riconoscibili canoni postmoderni (Ces.), viene la tentazione di chiamare in causa categorie critiche proprie di altri periodi storici (e applicate a testi del tutto differenti), per chiedersi se ci troviamo di fronte a un puro e semplice divertissement o se questa considerevole cifra d'inclusività risponda, per adottare una formula cara a Giacomo Debenedetti (Deb.), a un criterio di «necessità». Tradurre fedelmente il testo di una e-mail o la scritta che appare su un monitor, sforzandosi di riprodurne perfino la veste grafica, rappresenta un passaggio obbligato per veicolare informazioni che sfuggirebbero a una semplice parafrasi? È funzionale a un accrescimento cognitivo? Ci immette in prospettive altrimenti inesplorabili?

La risposta all'ultima domanda sembra essere affermativa. In effetti, la riproduzione mimetica degli inserti extraletterari rende meglio decifrabili le coordinate del mondo che Massaron descrive: una landa sovraffollata di esseri dallo sguardo catatonico o svuotato di ogni umanità, che non si discosta molto da quello di un display. Ma, una volta assolta questa funzione - il che, beninteso, non è affatto poco -, l'attenzione mercuriale e vorace dell'autore si sposta su altro, rifiutandosi di scavare, d'indagare ulteriormente nella psiche di quel personaggio che pure aveva così ben fissato nei suoi tratti essenziali. Alla forte spinta centrifuga imposta alla pagina corrisponde, insomma, assai di rado un movimento centripeto atto a farci scoprire un oltre, o quanto meno a farcelo intravedere.

Una significativa eccezione è forse costituita dal suo più recente lavoro: Ruggine (Rg), edito da Einaudi nel 2005. Costruito sul canovaccio del racconto antologizzato nove anni prima in Gioventù Cannibale - sono ripresi luoghi, personaggi e certi moduli espressivi di matrice approssimativamente definibile come "neorealista" - il romanzo segna, pur con qualche incertezza, l'ingresso della scrittura massaroniana in una fase più matura, caratterizzata da una maggior attenzione alla psicologia dei personaggi e da una mutata attitudine sperimentale, che si orienta ora verso la sovrapposizione dei piani diegetici e delle voci narranti.

Permane comunque uno degli elementi più riconoscibili dello stile di Massaron: la presenza della e-mail. Di questa forma l'autore sembra voler scoprire e sfruttare l'intera gamma delle potenzialità: l'agilità, l'immediatezza e, ancora una volta, l'inclusività, che gli consente, attraverso l'espediente degli allegati, di inserire dei brani di racconto che confondono ulteriormente i piani diegetici. A essa viene poi affidato il compito di innescare la vicenda: in seguito alla casuale lettura di una e-mail indi- 
rizzata a una rivista, Sandro ritrova una compagna d'infanzia e avvia con lei una corrispondenza; ma ecco che, proprio quando sembra definitivamente indirizzata verso il modello del romanzo epistolare, la narrazione compie una brusca inversione di rotta e abbandona le missive telematiche per inseguire il flusso di ricordi che travolge il protagonista. E con lui, l'intera struttura del testo, fino a quel momento rigorosa, che viene quasi del tutto scardinata, a favore di una rievocazione anarchica e fluida che resta - a mio avviso - quanto di meglio Massaron abbia mai scritto.

Messa da parte la sua debordante tecnofilia, egli si concede qui senza riserve alla scrittura - intesa, nel senso più partigiano del termine, come strumento conoscitivo per antonomasia - e, così facendo, finisce inevitabilmente con lo scalfire la superficie e sfiorare l'intima essenza dei suoi personaggi, se non della sua stessa vocazione letteraria. Il momento di grazia svanisce con i vaneggiamenti del Dottorboldini - tentativo piuttosto goffo di sondare la psiche di un pedofilo omicida - che ci riportano nell'ambito dello sperimentalismo più velleitario; ma finché dura, per quelle quarantacinquanta pagine di narrazione "pura", scaturite dall "impuro" espediente della posta elettronica, si ha la sensazione che la pagina scritta abbia trionfato e si è quasi tentati di farsi cullare dall'illusione che una e-mail possa ancora diventare la madeleine. 


\section{Bibliografia essenziale*}

(AMI) Nove Aldo, Amore mio infinito, Torino, Einaudi, 2000.

(Bal., I97I) Balestrini Nanni, Vogliamo tutto, Milano, Feltrinelli, I97I.

(Bal., I980) —, Blackout, Milano, Feltrinelli, I980.

(Bal., I994) -, I Furiosi, Milano, Bompiani, I994.

(Bal., 200I) —, La violenza illustrata; seguita da Blackout, Roma, DeriveApprodi, 200I.

(Cas.) CASAdei Alberto, Stile e tradizione nel romanzo italiano contemporaneo, Bologna, Il Mulino, 2007.

(CC) Veronesi Sandro, Caos calmo, Milano, Bompiani, 2005.

(CDC) Ammaniti Niccolò, Come Dio comanda, Milano, Mondadori, 2006.

(Ces.) Ceserani Remo, Raccontare il postmoderno, Torino, Bollati Boringhieri, 1997.

(Dc) Massaron Stefano, Doppioclic, Milano, Disney, $200 \mathrm{I}$.

(Deb.) Debenedetti Giacomo, Il romanzo del Novecento. Quaderni inediti, Milano, Garzanti, I97I.

(Eco) Eco Umberto, Apocalittici e integrati. Comunicazioni di massa e teorie della cultura di massa, Milano, Bompiani, 1964.

(FP) Veronesi Sandro, La forza del passato, Milano, Bompiani, 2000.

(Gab.) Gaber Gaber, C’è un'aria, in Io non mi sento italiano, album del 2003.

(GP) Mazzucco Melania, Un giorno perfetto, Milano, Rizzoli, 2005.

(Gr) Massaron Stefano, Graffiti, Roma, Adnkronos, 1998.

(IN) Ammaniti Niccolò, Io non ho paura, Torino, Einaudi, 200 I.

(KK) Massaron Stefano, King of Kings, Milano, Addictions, 200I.

(LN) - Lezioni Notturne, Bologna, Granata Press, 1994.

(Lup.) Luperini Romano, La fine del postmoderno, Napoli, Guida, 2005 .

(MCR) Nove Aldo, Mi chiamo Roberta, ho 40 anni, guadagno 250 euro al mese, Torino, Einaudi, 2006.

(PI) Massaron Stefano [sotto lo pseudonimo di Steven Farmer], «La prole immonda», in Maniac, n. I, I99I.

* Per una panoramica piuttosto completa e dettagliata della più recente narrativa italiana, si rimanda a Elisabetta Mondello, In principio fu Tondelli. Letteratura, merci, televisione nella narrativa degli anni novanta, Milano, Il Saggiatore, 2007. 
(Raj.) Rajewsky Irina, Intermediales Erzählen in der italienischen Literatur der Postmoderne, Tübingen, Francke, 2002.

(Ren.) Renello Gian Paolo, «Guida alla lettura», in Nanni Balestrini, La violenza illustrata. Seguita da Blackout, Roma, DeriveApprodi, 200I.

(Rg) Massaron Stefano, Ruggine, Torino, Einaudi, 2005.

(Rm) —, «Il rumore», in Daniele Brolli (a cura di), Gioventù Cannibale. La prima antologia dell'orrore estremo, Torino, Einaudi, I996, pp. I25-I52.

(Sim.) Simonetti Gianluigi, «Sul romanzo italiano di oggi. Nuclei tematici e costanti figurali», in Contemporanea, n. 4, 2006, pp. 55-8I.

(Sin.) Sinibaldi Marino, Pulp. La letteratura nell'era della simultaneità, Roma, Donizelli, 1997.

(SW) Nove Aldo, Superwoobinda, Torino, Einaudi, 1998.

(Ur.) Uricchio William, «La place de la télévision dans l'horizon d'attente du XIx ${ }^{e}$ siècle», in G. Delavaud (dir.), Dossiers de l'Audiovisuel. Un siècle de télévision. Anticipation, utopie, prospective, n. II2, novembre-décembre 2003, pp. 19-22. 
